\title{
Tangence
}

\section{« Le plus triste de la fin du monde, c'est peut-être que personne ne pourra la raconter "}

\section{Simon Harel}

Numéro 42, décembre 1993

Le récit de soi

URI : https://id.erudit.org/iderudit/025788ar

DOI : https://doi.org/10.7202/025788ar

Aller au sommaire du numéro

Éditeur(s)

Tangence

ISSN

0226-9554 (imprimé)

1710-0305 (numérique)

Découvrir la revue

Citer cet article

Harel, S. (1993). « Le plus triste de la fin du monde, c'est peut-être que personne ne pourra la raconter ». Tangence, (42), 49-67. https://doi.org/10.7202/025788ar d'utilisation que vous pouvez consulter en ligne.

https://apropos.erudit.org/fr/usagers/politique-dutilisation/ 


\section{«Le plus triste de la fin du monde, c'est peut- être que personne ne pourra la raconter " 1 Simon Harel}

\section{Le récit de soi}

L'autobiographie serait-elle une tentative de "survie" psychique dont l'enjeu est de mettre à distance une référentialité obsédante pour laquelle il s'agit précisément de trouver une explication satisfaisante? On peut le penser, du moins chez Leiris, puisque l'autobiographie correspond à une tentative de préhension existentielle qui acquiert une portée structurante. Réparer, par le recours à la fiction, consistera donc à structurer les cadres d'une énigme pour mieux offrir une réponse qui puisse donner signification au récit. Ainsi, dans "Mors", les souvenirs d'enfance du jeune Leiris coïncident avec l'énigme du silence paternel face à un bruit - à une signification - d'origine inconnue. Ce silence du père tiendra lieu de prétexte narratif qui inaugure une réflexion sur les divers avatars de l'absence. La mort est ici omniprésente de même que la "morsure" cannibalique qui fait de l'espace du dehors un monde angoissant, dévorateur.

Le titre même du premier chapitre de Fourbis est en effet évocateur: "Mors". Les relais associatifs sont multiples et se prêtent bien au jeu de la tresse dont Leiris a fait une des conditions de l'élaboration créatrice. Que lisons-nous?

[...] je vois mal pourquoi, au train dont $j y$ vais, je me retiendrais d'en appeler, par exemple, aux mandibules pour justifier, à l'aide de ce nouveau chaînon venu par la voie torse de la rime, le passage de l'insecte au somnambule - lui-même rattaché au travailleur sous-marin qu'est le scaphandrier, puis au monstre tombé d'une autre planète - et renforcer ainsi le lien un peu trop lâche qui s'est établi entre la bestiole tardivement susurrante et le dormeur éveillé, à partir de l'idée de solitaire d'un monde étrange (ou isolé insolite) en noctume intrusion. "Mandibules", plus que "gueule" ou que "mâchoire" (si fermement en place dans la vie journalière), ne recèle-t-il pas un

1 Michel Leiris, Joumal, 1922-1989, Paris, Gallimard, NRF, 1992, p. 626. 
50

danger singulier, comme la bouche charbonneuse que je croyais reconnaître ${ }^{2}$.

Il y a bien, dans ce passage, une description particulièrement réussie du rituel incorporant qui est au cœur du projet autobiographique. Le passage de mort à trépas, "d'obscurité à sommeil, de banlieue à désert, d'oubli à Zuyderzée, d'insecte à somnambule " ${ }^{3}$ est sans cesse répété. On peut donc parler à cette occasion d'un maintien tensif dont le caractère oscillatoire est clairement affirmé.

Ce qu'indique "Mors" de Michel Leiris, c'est en fait la toutepuissance du récit. Ici prévaut un souci de maîtrise dont le fantasme principal est certes la tentative de colmater une perte que l'affect dépressif signe clairement. Le récit est en effet dans l'œuvre de Leiris le marqueur d'une tension. Il peut surmonter la détresse qu'engendrerait une perception trop soudaine du manque. Leiris écrit:

Il me faut donc remonter. Pas seulement de ce gouffre métaphorique: le sommeil dans lequel envie et faculté d'écrire peuvent s'enterrer pour une durée illimitée, mais d'un trou beaucoup plus positif: celui qu'un livre, dès l'instant qu'il a subi son avatar définitif en étant publié, creuse au plus intime de nous $[\ldots] .4$

Le récit serait donc cet assemblage de signifiants qui permet de fuir une crypte mortifère. Le trou, auquel Leiris fait référence avec insistance, c'est d'abord cet affect dépressif qui fonde l'écriture. Il faut, en somme, qu'un signifiant chute pour que l'écriture advienne. Il faut qu'une déprise narcissique fasse du sujet un autre que soi pour que cette "durée illimitée" soit constatée. Sans doute est-ce que la traduction de la pensée semble chez Leiris l'enjeu même de l'interrogation sous-jacente au travail d'écriture. Que penser en effet de ce "gouffre métaphorique " qu'est le sommeil? Que penser de cet enterrement qui est associé à la perte du temps? Leiris, on l'a vu, parlera à ce sujet de "durée illimitée". Faut-il en conclure que l'écriture est justement ce qui permet une déprise? En somme, que l'articulation du signifiant dans l'écriture de Leiris, comme repère d'une chute, d'un laisser-aller (d'une

2 Michel Leiris, Fourbis. La règle du jeu II, Paris, Gallimard, 1955, p. 28.

3 Ibid.

4 Ibid., p. 8. 
acceptation de l'attachement comme discontinuité) inaugure cet affect dépressif: trou, béance qu'il faut constater? Et si ce trou d'autant plus angoissant qu'il survient suite à "l'accouchement" d'un livre - était précisément ce qui doit être comblé. Je cite sur ce point Michel Leiris:

Sensation de vide, non seulement parce que nous nous sommes "vidés", très exactement, de ce que nous avions au cour et dans la tête, mais parce que nous éprouvons avec acuité que [...] ce livre aura été pour nous un vrai geste dans le vide en comparaison de ce que nous avions espéré. ${ }^{5}$

On pourrait retenir à cet égard, sous l'égide de la métaphore incorporante qui soutient le récit, la figure d'une bouche à remplir de mots, d'un trou à combler, d'une perte à colmater sinon à réparer.

Écrire dans la perspective qui est ainsi adressée signifierait que l'écrivant propose un signifiant dont la valeur incorporante lui permettrait de remplir une angoisse-panique. Leiris écrira, toujours à propos de ce souvenir d'enfance qui inaugure la défaite de la fonction paternelle:

[...] il demeure qu'à un moment quelconque du temps selon lequel se fait et se défait ma vie $[\ldots .$.$] - vers moi cette voiture$ qui ne pouvait être tirée que par un cheval s'est avancée comme l'un des accessoires contribuant à produire un effet de peur, soit qu'elle ait fait matériellement partie du scénario réel, soit qu'en vertu de quelque affinité indécelable elle se soit, un beau jour, ajoutée à la réminiscence qui en était le reflet plus ou moins déformé. ${ }^{6}$

Leiris ici est explicite. Cette voiture dont l'apparition fut la source d'une violente angoisse exista bien un jour. Mais la porosité de l'imaginaire ne permet pas de dire où loge précisément ce souvenir dont Leiris peut souligner tout au plus qu'il s'agit "matériellement" d'un scénario réel. À cet égard, le procès mémoriel, que l'on retrouve mis en cuvre dans "Mors", privilégie les solutions de la continuité, de la déhiscence, de la fragmentation. L'événement remémoré est-il véridique? S'agit-il au contraire d'une élaboration associative construite de toute pièce à la manière d'un souvenir-écran? La dernière hypothèse serait d'autant 
plus plausible que la représentation de la scène théâtrale, entrevue comme insertion topique d'un espace à découvrir, est omniprésente dans Fourbis. Je cite un passage introductif de "Mors":

"Rideau de nuages".

J'ai toujours réagi fortement à cette expression - évocatrice d'un espace scénique élargi aux proportions de l'infini quand je la lisais, marquant la césure entre deux tableaux, dans le livret d'une cuvre de Wagner [...] figure d'une nébulosité tirée devant la vue comme pour signifier doublement l'interruption de la durée: rideau qu'elle est, d'abord, de toile peinte ou de gazes presque transparentes superposées comme les volants d'une jupe de tulle; image vague, ensuite, suggérant le chaos qui est la négation du monde temporel et spatial où règnent nos coordonnées. ${ }^{7}$

Ce "rideau de nuages" auquel Leiris fait référence est bien sûr la négation de "l'écran de néant" ( $c f$. Le point cardinal) dont la mort semble être la plus juste représentation. Chez Leiris, ce "rideau de nuages" suppose "l'interruption de la durée": artifice nécessaire puisqu'il justifie la transition entre deux scènes. Mais ce "rideau de nuages " est d'une quasi transparence qui suggère à la fois "les volants d'une jupe de tulle" et "le chaos qui est la négation du monde temporel.

En somme la problématique de l'inscription sensorielle dans "Mors" obéit à cette non-distinction angoissante. La scène théâtrale est à la fois ce lieu qui permet d'oblitérer la présence angoissante de la mort. Le rideau apparaîtra alors comme un obstacle qui met à l'écart, qui protège, somme toute, qui fait obstacle. Mais la transparence de l'artifice ne peut garantir la mise à l'écart de toute effraction pulsionnelle. C'est donc sous un mode éminemment traumatique que se constitue la représentation mémorielle dans "Mors". Leiris ajoutera:

Passage d'un fiacre, donc, ni insecte ni scaphandre; fiacre seulement que tire un quadrupède à longue tête dont la bouche, par l'intermédiaire du mors auquel sont attachées les guides, est relié aux mains d'un cocher et dont les flanc sont pris entre deux brancards, de ces brancards qui se brisent si souvent quand le quadrupède tombe et s'agite convulsivement sur le pavé, scandale soudain parce qu'éclatement public de la tragédie, telle la chute de quelqu'un que frappe le haut mal ou 
de celui dont le sang, toute vergogne anéantie à la suite de quelque accident, se répand brusquement au dehors en une horrible excrétion. ${ }^{8}$

Ce passage de "Mors" m'apparaît particulièrement significatif. Il y est question, en effet, d'une mort violente qui vient posséder le sujet sous la forme d'une hémorragie. La blessure est patente. Elle ne peut plus être colmatée. Faut-il voir dans cette figuration du cheval l'équivalent d'une mort dont l'instinctualité est clairement affichée? Peut-être, d'autant que cet effroi est vu sous la forme socialisée de: "La crainte que l'enfant bourgeois a de l'ivrogne et surtout celle de l'ivrogne pauvre, du pauvre tout uniment dès qu'il se déchaîne tant soi peu, rejette la bienséance que l'ordre des gens aisés lui impose et zigzague sur le trottoir [...]. "9 À la faveur de ce passage, c'est bien d'une perte de mầtrise dont il est question. Maîtrise de soi sous la forme d'un maintien corporel que "l'ivrogne pauvre" ne peut revendiquer. Maîtrise d'un monde interne sous la forme de cette hémorragie qui amène le narrateur-autobiographie à constater l'horreur de la blessure qui est ici associée à la mort. Cette blessure interne, elle s'apparente à une "horrible excrétion" dont on peut constater le caractère angoissant. Chez Leiris, notamment dans "Mors", c'est la représentation de l'autre - sous l'aspect d'une figure qui recourt au modèle de l'identification projective - qui permet de construire, même fragmentairement, le tableau d'une autobiographie pour laquelle le deuil est un motif prédominant. C'est par la voie de l'expulsion que se constitue l'appréhension de l'Autre. Il reste néanmoins à se demander quelle est la nature de cette perte, selon quelles modalités psychiques est-elle mise en scène? Sans doute faudrait-il parler à propos de ce passage de Fourbis de l'introjection de la mort elle-même sous la forme d'un originaire problématique. C'est, à mon sens, l'enjeu circonsctit par ce récit. Nombreux sont les passages et les descriptions qui tentent de personnaliser à outrance les manifestations de l'angoisse de mort du jeune Leiris. Comme si une logique causale pouvait rendre compte, même à la faveur du motif de la tresse, de la structuration de l'événement psychique. Et, du même coup, donner nom et forme à cette angoisse de disparition qui structure le projet autobiographique. Pourtant Leiris n'est pas dupe et mentionne à 
plusieurs reprises la valeur narrative de cette reconstruction mémorielle qui ne se contente pas d'ordonner le récit, mais qui lui associe une valeur opératoire. Raconter, ce n'est pas seulement agencer des séquences, c'est surtout, point essentiel, trouver l'intervalle exact entre les séquences mêmes. En somme, l'on rencontre dans ce texte de Leiris les interrogations qui parcourent des écrits antérieurs, entre autres L'âge d'homme. S'il y a bien un dédicataire qui, chez Leiris, inaugure un acte de lecture qui fonde fantasmatiquement le récit, il n'en reste pas moins que celui-ci varie au gré des investissements figuratifs mis en œuvre.

Je crois que nous touchons là un point essentiel de l'écriture de "Mors". Ici l'emphase figurative est volontairement désavouée. Il n'y a plus d'autre. Il n'y a que le constat de la perte de l'Autre. Leiris écrit dans "Mors":

Quelque chose qui, de ce fait, ne peut être entendu de nous que comme un bruit de glas: sans commune mesure avec notre propre vie (puisque cette chose, que nous la disions insecte ou voiture à cheval, demeure au fond impénétrable étant donné l'ignorance où nous sommes de ce à quoi elle est occupée), indépendante de nous comme elle est indépendante du reste (de tous ces êtres qui, en apparence, sont endormis alors qu'elle veille) n'exprime-t-elle pas de manière tangible une permanence imperturbable qui est celle même du cours des choses, soit l'un des aspects de la mort les moins aisés à considérer sans trembler, à savoir que notre fin a toutes chances de n'être pas fin du monde, mais seulement fin se limitant - injustement, semblera-t-il toujours — à nous ${ }^{10}$

N'est-ce pas justement cet intervalle - ce bruit de glas - qui signe la présence impersonnelle de la mort? Ne s'agirait-il pas dès lors d'un dédicataire dont l'introjection est impossible?

\section{La défaillance autobiographique}

On sait qu'Aurora de Leiris "figurait" une réalité en voie d'effondrement puisque le personnage féminin n'existait qu'au prix d'une insupportable mise à mort. Nul doute que l'omnipotence du dédicataire féminin était ici contestée avec une violence extrême. Le propos de L'âge d'bomme est certes différent car on 
peut entrevoir à cette occasion la persistance d'un "roman familial" qui fonde la constitution du récit et qui situe le projet autobiographique d'un énonciateur manifeste. Si l'on retrouve dans l'âge d'bomme une élaboration fragmentaire qui recourt notamment au procédé du photo-montage, c'est sur fond de surdétermination défensive que le récit se constitue. Les descriptions de traumatismes subis sont encore soumises à une esthétique qui fait du regard l'enjeu d'une véracité à localiser. Les blessures représentent autant de symptômes qu'il faut violemment déchiffrer. En témoigne entre autres le récit des infirmités et déficiences qui compose une des trames majeures de L'âge d'bomme. L'on rencontre en fait, avec l'âge d'bomme, l'aveu d'une violence originaire qui est marquée sur le corps du sujet. Le ruban au cou d'Olympia représentera, sous une forme beaucoup plus élaborée, le complément de L'âge d'bomme. Le regard permet de donner sens à l'invisible:

Couper les mains, couper les pieds, voire couper les oreilles ou le nez, peut se faire sans que cela entraine la mort. Mais pas couper le cou, trancher le pédoncule relativement gracile qui relie la tête au torse. Particulièrement émouvant [...] sera donc, l'ornement du cou, et plus encore si, ruban d'un souple tissu et non collier aux durs éléments minéraux, il apparaît propre à masquer et discrètement réparer une légère mais cruelle blessure qui aurait entamée le cou. ${ }^{11}$

La découpe de l'espace ainsi instaurée fonde un récit qui vaut par la détermination de ses frontières. La blessure est le signe d'une défaillance qu'il s'agit de réparer. Sans doute retrouvons-nous chez Leiris, autour du motif de la blessure, la mise en scène de l'identification projective: processus psychique permettant à la fois d'expulser (d'attribuer à l'Autre) ce qui est perçu comme une souffrance psychique insoutenable; et de réintrojecter - sous une forme modifiée - cette destructivité pulsionnelle.

L'âge d'bomme, mais aussi La règle du jeu, témoignent du caractère réitératif de la tresse chez Leiris: surdétermination signifiante qui donne au narrateur la latitude d'associer librement, en s'assurant néanmoins d'un "lien signifiant " qui permet aux champs sémantiques de s'agglomérer. Sans doute est-ce que le motif de la tresse recoupe une élaboration secondarisée qui travaille,

11 Michel Leiris, Le ruban au cou d'Olympia, Paris, Gallimard, 1981, p. 268. 
56

réélabore des fragments afin de leur octroyer une unité. Ce motif de la tresse, s'il est encore présent dans "Mors", me semble néanmoins être abordé sous un tout autre angle. La tresse révèle, à la fin du parcours élaboratif, un écran vide, une image du corps absente, une identité lacunaire puisque la "perte" de l'Autre (en l'occurrence la mort du père) ne peut faire l'objet d'aucune représentation. Il s'agit là d'une innovation particulièrement forte dans l'écriture de Michel Leiris. Le symptôme n'est plus la représentation d'un substrat psychique ou physiologique. Il faudrait plutôt parler de la dynamisation de cette absence qui n'est plus seulement pétrifiante, mais qui permet de fonder un intervalle (une scansion de la durée) par le biais du récit. Peut-être retrouvons-nous dans "Mors" à cette occasion une modification substantielle de la posture narrative. Alors que L'âge d'bomme valorise une élaboration panoptique du discours autobiographique (il faut voir pour comprendre, localiser pour déterminer le trauma qui donne sa force au "récit de soi"), "Mors" inaugure une inscription temporelle de l'autobiographie. L'espace n'est plus uniquement ce qui découpe et permet de fonder des repères précis qui structurent le discours du narrateur. Le modèle autobiographique, s'il obéit encore à une modélisation spatiale, n'en est pas moins justifié par une temporalité qu'il sera impossible de réparer. Là se situe à mon avis l'avancée du projet leirisien. Il faudrait alors se demander si cette impossible réparation, à laquelle je faisais référence précédemment, n'est pas concernée au premier chef par la hantise de la mort et de l'intervalle définitif qu'elle instaure. La réparation serait confrontée de manière particulièrement radicale à ce hors-pensée qui me semble être ici une caractéristique du processus créatif. Faut-il alors imaginer que le projet autobiographique donne forme à l'impensé qu'est la mort? Il me semble que la perspective envisagée est plus complexe puisque l'intervalle narratif, dans "Mors", demeure associé à un entendu pour lequel nulle vision ne peut offrir d'attestation: "Quelque chose qui, de ce fait, ne peut être entendu de nous que comme un bruit de glas: sans commune mesure avec notre propre vie. " 12

Que signifie ce passage énigmatique de "Mors"? Sans doute d'abord l'aveu d'une déhiscence entre l'audition et le regard. Cette non-coïncidence sensorielle est associée à l'omnipotence

12 Michel Leiris, Fourbis. La règle du jeu II, op. cit., 1955, p. 31. 
angoissante de la mort. Le glas serait alors ce fragment de sens, échappant à la destruction, qui pourrait permettre de construire, de justifier une élaboration discursive ténue, qui puisse échapper à la fragmentation. C'est ainsi que nous sommes autorisés à parler de restitution de la chose entendue dans "Mors", processus qui me semble correspondre au motif de la réparation que j'ai abordé précédemment. Cette non-coincidence sensorielle est paradoxalement une pensée de la discontinuité qui fonde le récit. Que le bruit entendu ne soit pas attesté par le porte-parole paternel, voilà qui introduit l'angoisse de mort pour laquelle, selon le discours tenu par Leiris, tout ne pourrait qu'être silencieux. Il faut donc rompre ce silence, l'envelopper de mots protecteurs qui permettent de mettre à distance cette "terreur sans nom" dont Bion a judicieusement défini la structure. C'est en effet la crainte d'un bruit sans référent qui hante l'écriture de Leiris. Comme si le fragment signifiant entendu était, c'est ce qu'écrira Leiris, indépendant de nous, sans commune mesure avec l'existence. La seule solution offerte est ce repli corporel (et narcissique) qui permet de contrer la menace d'un anéantissement. Leiris écrira, toujours dans "Mors":

Non seulement, lorsque je suis inquiet, j'ai tendance à me replier, à me terrer (n'être rien, afin que la mort m'oublie, et peut-être est-ce aussi pour cela que les alarmes de notre époque m'éloigneraient de la poésie plutôt qu'elles ne m'en ouvriraient les sources, déjà si peu généreuses ordinairement à mon égard), non seulement j'éprouve un besoin de repli qui s'oppose à tout mouvement d'effusion [... . . ${ }^{13}$

Ce repli est bien sûr l'aveu d'un retrait narcissique (le refus explicite de la procréation en étant à mon sens la manifestation première). Mais ce repli permet de survivre. Or la problématique de la survie s'avère fondamentale face à une ouvre où dominent les motifs de l'attachement et de la perte de l'objet. Le narrateurautobiographe nous rappelle somme toute qu'il ne peut s'attacher à une réalité qui lui échappe, dont les signifiants sont énigmatiques. Qu'il ne peut donner sens à un bruit dont le référent demeure lacunaire. Se replier pour mieux survivre supposerait la mise à l'écart de toute souffrance psychique intolérable. Sans doute l'écriture de "Mors" est-elle concernée au plus haut point par cette auto-perception de la psyché.

13 Ibid., p. 65. 
58

Ce n'est pas donc pas seulement la mort "réelle" qui est redoutée, mais son correspondant psychique qui est au cœur du projet autobiographique leirisien: l'angoisse de disparition. Si Leiris situe une énigme - ainsi, le silence angoissant du père et sa "valeur traumatique " au moment de la petite enfance - - c'est du même coup afin d'octroyer à la trame narrative une portée réparatrice. Il serait insupportable en effet que le récit ne puisse donner de "nom" à cette absence de représentations. Il serait intolérable que cette angoisse ne s'insère pas dans le cadre d'une trame langagière où prévaut l'intersubjectivité d'un dédicataire dont on puisse être assuré qu'il soit en mesure de recevoir l'œuvre. Le processus réparateur est envisageable dans l'œuvre de Leiris du fait de la persistance de l'Autre qui peut bien être étrangement distant, à moins qu'il ne se révèle d'une proximité étouffante. Peu importent les modalités de cette relation à l'objet, ce dernier existe et fait valoir le respect qui lui est dû.

Sous cet aspect, on pourrait envisager dans l'œuvre de Leiris la mise en scène d'une autobiographie anthropophage où le désir de l'objet est tel que celui-ci se doit d'être intégralement possédé. Si ce fantasme fusionnel accompagne l'élaboration de l'écriture leirisienne, il n'en reste pas moins que le moment de non-distinction - entre soi et autrui - reste provisoire. Ainsi l'écriture d'Aurora représente bien le désir achevé de produire une jouissance problématique suite à la contemplation d'une féminité mise à nue et démembrée; désir qui est consécutif à la mise en scène de l'identification projective qui structure, chez Leiris, le projet narratif. Cependant, tel est l'enjeu du récit autobiographique, il faudra malgré tout proposer une "représentation" fidèle de l'Autre. En témoigne sur ce point le projet mis en œuvre dans Le ruban au cou d'Olympia puisque la contemplation de la nudité féminine doit donner quelques réponses à cette énigme qu'est l'étrange absence de l'Autre. Voilà en somme ce qui permet de constituer la spécificité du dédicataire féminin dans l'écriture leirisienne. Si l'interrogation créatrice prend à témoin l'Autre qui devient alors le révélateur du sens caché de l'autobiographie, c'est bien parce que ce dédicataire acquiert une fonction contenante.

Le motif esthétique du Ruban au cou d'Olympia est à cet égard déterminant. Le corps féminin est entrevu à la manière d'un théâtre qui révèle une crypte matricielle. L'écriture a donc pour fonction première de restituer un cadre figuratif qui puisse donner sens à ce fragment que représente justement le ruban au cou 
d'Olympia. Il faudrait parler, à propos de cette activité restitutrice, de l'intégration - sous forme de greffe - d'une relique qui fait lien avec le corps d'Olympia. En fait, la reconstitution du corps d'Olympia permet de fonder, par le biais de l'écriture, un Moipeau (ou encore une enveloppe psychique) qui dresse le portrait du sujet narrateur-autobiographe. J'ai noté plus tôt la pertinence de l'activité représentative dans l'œuvre de Leiris. Le derme est une surface qui fait l'objet d'une inscription sensorielle dont l'écriture est l'instrument privilégié. Les motifs de la béance, de la perte révèlent un processus de symbolisation qui recoupe, à mon sens, une interrogation sur le statut de l'originaire et de la transmission. Cette interrogation chez Leiris n'est pas gratuite. Elle permet de fonder l'œuvre, mais à la faveur d'un mouvement d'anticipation qui inscrit l'angoisse de disparition comme figure centrale. Écrire pour ne pas mourir; ou encore, écrire pour précéder la mort et ainsi en offrir une symbolisation: telle est sans doute la caractéristique de cette écriture dont l'élaboration formelle est particulièrement révélatrice. Quiconque lit Leiris est d'abord surpris par ce lent enveloppement produit par une écriture sinueuse qui fait place à une effraction pulsionnelle particulièrement violente. Les poèmes de Leiris (notamment dans Haut mal suivi de Autres lancers) rendent compte de cette problématique. Le corps y est présenté comme un objet minéralisé meurtrissant le sujet qui habite cette "peau métallique" dont toute sensorialité est évacuée. Il y a bien mise en scène d'une blessure dans ces poèmes qui inscrivent la crainte d'un déchirement de l'enveloppe corporelle. Mais cette blessure est externe. Elle écorche un derme dont la dureté - et l'imperméabilité métallisée - suspend toute effraction.

Or l'œuvre ultérieure de Leiris me semble correspondre (sous l'angle d'une métapsychologie de l'acte d'écriture) à l'activation d'une effraction pulsionnelle. La violence impersonnelle qui torture le sujet m'apparaît être une caractéristique de Haut mal. Pr. contre, L'âge d'homme adopte un point de vue narratif introspectif. L'autobiographie fait appel à une structure diégétique qui privilégie la scène théâtrale. Le regard compose une scène omniprésente qui sous-entend un contemplateur indiscret. Leiris mentionne de plus, dès les premières pages de L'âge d'bomme, que la composition du récit emprunte au modèle du photomontage. C'est en effet le collage qui permet la concaténation des divers scénarios qui composent l'âge d'bomme. L'énonciation des traumatismes de l'enfance suppose une anamnèse grâce à la- 
quelle il sera possible de réintroduire la trame d'une mémoire défaillante. Le narrateur de L'âge d'bomme fait appel à une mémoire dont le caractère omnipotent est clairement affirmé. Il ne s'agit pas simplement de structurer les motifs de la passéité. L'impératif est ici catégorique: "je me souviens" (je dois me souvenir), telle est la torsion mémorielle qui oriente l'écriture de ce récit.

Dans L'âge d'bomme, l'autoportrait que propose le narrateur est circonscrit de façon très volontaire. Ce dernier doit rendre compte d'une expérience traumatique (en témoigne le scénario de la gorge coupée) qui pourrait être abréagie grâce au discours narratif. L'énonciation autobiographique (même lorsqu'elle emprunte le modèle pictural de l'autoportrait qui offre l'attestation imagée d'une présence) se veut remémoration fidèle. Si le motif de la blessure est insistant dans l'œuvre de Leiris, c'est peut-être parce que cette première représentation d'une coupure (d'une perte) fait de l'écriture l'enjeu incessamment recommencé d'un travail du deuil. Reconnaître la blessure (même sous la forme de l'identification projective qui encapsule dans l'Autre féminin cette blessure traumatique qui tenaille le narrateur), c'est au moins avouer (dans une perspective autobiographique qui conjugue culpabilité et jouissance de la confession) l'existence d'un intervalle, d'une séparation qui pourrait faire sens. À ce titre, l'énonciation mélancolique chez Leiris, qui peut être perçue dans un premier temps comme perte de l'objet, implique une blessure narcissique qui affecte douloureusement le Moi. Il y a en somme un deuil - toujours répété dans l'écriture - qui affecte - et fait chuter - la signification dans une temporalité non advenue qui devra faire l'objet d'une reprise. Ainsi se constitue la torsion, j'ajouterais le caractère tortionnaire de l'écriture leirisienne. C'est que l'aveu est impératif. La quête de soi trace le parcours d'une écriture. Le traumatisme et sa mise en scène figurative - permettent de situer un "originaire" à partir duquel la narration pourra prendre forme. En somme, la mélancolie - sous sa forme de magie incorporante peut laisser advenir le travail du deuil dont l'affect dépressif me semble particulièrement significatif dans l'œuvre de Michel Leiris.

\section{«Les maisons vides »}

Un passage de "Pierres pour Giacometti", bien qu'il fasse référence au travail du sculpteur, est révélateur de cette violente effraction qui caractérise le projet autobiographique leirisien: 
Gratter jusqu'à l'os, jusqu'à l'indestructible. Ou bien - inversement - ajouter de l'espace alors qu'on semble vouloir éliminer quelques onces de matière. [...] Faire, perfectionner, défaire, puis refaire, reperfectionner, redéfaire... par désir de rigueur jusqu'au moment où, les circonstances imposant d'en finir, la chose est arrêtée telle quelle, à ce désir de rigueur s'étant simplement substituée la soumission à un état de fait, comme à une loi succède une autre loi, ${ }^{14}$

Gratter jusqu'à l'os, voilà bien ce qui caractérise un désir obstiné qui est à la source du processus créateur. Non pas seulement mettre à nu, ainsi que Leiris le revendiqua à de nombreuses reprises, mais plus fondamentalement "révéler" cette ombre squelettique à partir de laquelle une "représentation" de la psyché et de l'inscription corporelle - est rendue possible. Nul doute que l'on retrouve aussi, de façon radicale, cette quête d'une éventuelle réparation de soi par le biais du processus créateur. J'ai déjà souligné ce qu'impliquait un tel fantasme de reconstruction: le désir d'abolir la mort et de s'instituer survivant face à son "double" périssable. La notion de réparation, parce qu'elle implique ici la reconnaissance du dédicataire maternel, s'avère d'autant plus pertinente qu'elle nous amène à prendre en considération la défaillance qui, dans l'œuvre de Leiris, lui fit construire et reconstruire de manière incessante cette "carapace musculaire". L'on sait, par le Joumal, qui fut publié après la mort de Leiris, que la dernière campagne d'écriture portait comme titre provisoire Les maisons vides. Belle manière, dira-t-on, d'habiter l'écriture et de rejeter le spectre de la mort. Ces "maisons vides", elles ne sont pas sans rappeler ce corps fragile, vieillissant dont Leiris, pressentant sa "fin", nous disait, par le récit, la nécessité de le vitaliser. Le récit tenant lieu, par la signification temporelle qu'il instaure, de possible reconstruction mémorielle. L'acte d'écrire, tel qu'entendu par Leiris, ne serait donc pas si éloigné du jeu avec la matière que l'on retrouve dans l'œuvre de Giacometti.

Quand Leiris indique à propos du sculpteur certe sorte de volonté forcenée qui consiste à " [...] faire, perfectionner, défaire, puis refaire, reperfectionner, redéfaire", il souligne bien la part d'indécision et de malléabilité figurative qui entoure l'acte même de créer. Mais une telle proposition, ainsi que Leiris se plaît à la

14 Michel Leiris, "Pierres pour Giacometti ", Brisées (1966), Paris, Gallimard, coll. "Folio/Essais", 1992, p. 174-175. 
formuler, possède d'autres résonances. Faire une œuvre dont la violence amène à épuiser la matière, gratter l'os jusqu'à la découverte de cette incarnation première qui s'avère indestructible, tel est le lot de cette fétichisation de l'image du corps que l'on retrouve dans l'œuvre de Leiris. C'est que le travail de Giacometti est perçu par Leiris de façon assez singulière. On peut y voir, d'une part, une esthétique de la fragmentation et de la reconstitution qui n'est pas si éloignée d'autres commentaires de Leiris, notamment envers les recherches d'André Masson. Cet acte qui consiste à détruire pour mieux reconstruire, il s'inscrit, d'autre part, au coeur d'un projet réparateur dont nous avons entrevu les modalités. Ainsi le formule Leiris: "À voir les espèces d'hécatombes auxquelles Giacometti s'est livré, je me suis parfois demandé si sculpter, pour lui, ne signifierait pas fabriquer quelque chose qu'on peut anéantir ensuite. "15 Que signifie en effet inscrire l'indestructible, pour paraphraser Leiris, alors que la création résiste à toutes les tentatives de désintégration? La réponse peut sans doute être trouvée dans l'intérêt assidu que Leiris témoigne envers l'œuvre de Giacometti. On notera que cette indestructibilité de l'objet de perspective (la sculpture chez Giacometti) n'est pas sans rappeler l'écorchement qui, toujours chez Leiris, permet la projection de soi sur une œuvre: essai désespéré afin de tromper - et de vaincre - la mort. L'autobiographie, ainsi que l'entrevoit Leiris, ne correspond-t-elle pas précisément à cette inscription topique qui institue la scansion de la durée pour mieux abolir l'idée même de la passéité.

À l'instar de Giacometti qui "[...] ajout[e] de l'espace alors qu'on semble vouloir éliminer quelques onces de matière", le projet autobiographique fait appel au "je dis": forme contemporaine de l'énonciation qui contrecarre le spectre de l'avoir-été. Il y a bien, à l'occasion de ce projet autobiographique, le souhait de transformer, selon les propres mots de Michel Leiris, l'angoisse en mélancolie. C'est Giacometti qui sculpte ces personnages qui ont toutes les apparences de sujets dont la "présence réelle" 16 se situe "entre le géologique et l'aérien"17. Faut-il parler à ce sujet d'une esthétique de la ruine qui ferait jouer la valeur commémorative de l'objet? Si tel était le cas, nous retrouverions dans cette

15 Ibid., p. 174.

16 Ibid., p. 175.

17 Ibid., p. 174. 
soudaine découverte du visage humain l'équivalent d'une indestructibilité primaire à laquelle il faut associer la perception du visage maternel. Ainsi le formulera Leiris à propos de Giacometti :

Quelque chose des ruines de Pompéi et des peintures murales demeurées fraîches malgré le vent, l'orage, les cendres. Dans les trouvailles telles qu'en font les archéologues convergent l'antiquité millénaire et la rupture fulgurante du temps: dévoilement brusque d'une figure dont, à jamais, se totalise le long passé. Quand à ses propres trouvailles, il semble que Giacometti - commandant à ses mains comme à des ouvriers qui procèdent à des fouilles - voudrait les extraire de son cerveau, armées de pied en cap. ${ }^{18}$

Cette trouvaille, semblable à celle des archéologues, comment ne pas la comparer à l'investigation de Norbert Hanold, amoureux fou de l'empreinte laissée par la Gradiva. On sait que Freud recourt à cette nouvelle de Jensen afin d'indiquer de quelle manière le fantasme ignore souverainement la distinction entre réalité et fiction. Peut-être faut-il ajouter que l'œuvre même de Giacometti, telle qu'elle est perçue par Leiris, souscrit à cette trouvaille du mouvement, de la vitesse, en somme de l'animation de l'inanimé. Au-delà d'une esthétique mortuaire qui cherche à recomposer le portrait du sujet disparu, cette jouissance forte, inscrite dans l'œuvre de Giacometti, laisse entendre que la "présence réelle" est à la fois pétrifiée et animée. La pétrification n'aura donc duré qu'un temps puisque la rétractation narcissique qui habite l'œuvre de Giacometti permet le surgissement du mouvement:

Peint, le bronze échappe plus aisément à la condition de sculpture que lorsqu'il a gardé son aspect métallique. Si L'bomme qui tombe est demeuré exclu de la série d'ouvres auxquelles Giacometti, en 1950, fit subir ce traitement, serait-ce parce qu'une chute coulée en bronze n'est jamais menacée de se figer en statue? ${ }^{19}$

La problématique autobiographique, abordée par Leiris, n'est pas tellement différente des projets qui sont attribués à Masson et à Giacometti. Il s'agit toujours de vitaliser le langage afin de lui attribuer un "visage" qui correspond, c'est mon hypothèse, à la figuration d'un dédicataire maternel. C'est en effet ce dernier qui 
64

peut assurer à l'auteur sa postérité bien qu'une telle entreprise soit envisagée avec un dédain sans équivoque. La mort de soi est en effet le signe indélébile de la disparition d'autrui. Il y a, au coeur de la démesure du projet autobiographique signé par Leiris, le souhait que la mémoire persiste malgré la disparition de l'énonciateur qui en est pourtant le porte-parole. Souhait étrange que cette postérité échappant à l'auteur car le "travail " du littéraire ne consiste-t-il pas justement à assurer cette commémoration culturelle? Le livre n'estil pas cet objet qui répare, par l'illusion vitale qu'il procure, l'anticipation de la mort? Il faudrait alors comprendre l'insistance autobiographique chez Leiris comme l'expression d'un maintien narcissique: "La mort ou le malheur qui nous frappera, par exemple, avant la livraison d'un costume commandé en toute innocence. "20 À quoi l'on peut ajouter, en puisant dans le Joumal: "Le plus triste de la fin du monde, c'est peut-être que personne ne pourra la raconter." 21 Que retenir de ces différentes affirmations dont celle-ci est peut-être la plus significative:

Le jeune Liégois qui est venu me voir au Musée de l'Homme et avec qui je suis rentré à pied, me dit qu'il y a peut-être une relation entre ma répugnance à procréer et mon incapacité d'inventer des personnages de roman. J'acquiesce, tout en lui disant que je n'y avais pas songé. Y réfléchissant ce matin, je pense que l'explication de ce rapport est bête comme chou: mon narcissisme, mon égocentrisme, qui m'empêche d'"enfanter. en quelque sens que ce soit (mettre quelqu'un d'autre au monde ou créer fictivement quelqu'un d'autre). ${ }^{22}$

Vitaliser le langage revient donc toujours chez Leiris à fétichiser l'instant présent, de peur que la scansion de la durée fasse intervenir l'anticipation angoissante de la mort. On perçoit le caractère paradoxal d'une telle proposition puisqu'elle contribue à dénier le dialogisme qui est à l'œuvre au sein même du discours autobiographique. Car parler du Livre, comme le fait Leiris, institue une position d'énonciation ubiquiste. Le narrateur est le seul à pouvoir témoigner du Grand Récit qu'il élabore patiemment. La mort du récit est le signe de l'effritement de l'Auteur ainsi que le souligne Michel Leiris dans son Journal. Ce maintien narcissique - qui recourt souvent d'ailleurs à la métaphore vesti-

20 Michel Leiris, Joumal, 1922-1989, Paris, Gallimard, NRF, 1992, p. 564.

21 Ibid, p. 626.

22 Ibid., p. 672. 
mentaire - n'est pas sans profondes résonances. Le caractère hautement narcissique du projet autobiographique provient très précisément de la confusion entretenue entre soi et autrui. Devenir soi-même cette pierre qui est la métaphore par excellence de l'inanimé, telle est l'angoisse dont la représentation tenaille l'écriture de Leiris. Cet effritement, à la fois craint et souhaité, correspond à cette certitude déjà énoncée dans "Mors": la disparition du narrateur ne signifie pas seulement le constat tragique ou désabusé de sa propre finitude. Plus fondamentalement, cette disparition est aussi le signe de l'effritement de la réalité externe. Ne pas pouvoir créer, ainsi que le font les romanciers, des personnages distincts, animés d'une vie propre, voilà ce qui explique - aux yeux de Leiris - la faillite singulière de l'autobiographie. Encore qu'il soit nécessaire de s'entendre sur la signification donnée à ce discours. Il ne s'agit pas de revendiquer, à propos de l'autobiographie, le caractère anecdotique d'un genre littéraire dont serait retenue la dimension confessionnelle. Nous avons déjà évoqué de quelle façon le discours de Leiris s'inscrit au cour d'une problématique du dévoilement et du "rachat " par l'écriture. Si cette réflexion fait l'objet de nombreuses mises en perspective, notamment dans le Joumal, il n'en reste pas moins que la "confession" est aux yeux de Leiris un simulacre qu'il convient d'aménager narrativement.

\section{La disparition du récit}

L'autobiographie est faillible du fait même du monologisme qu'elle instaure. Le maintien narcissique est une ruse discursive qui contribue à faire du narrateur l'Auteur dont dépendra la survie du récit. Le témoignage (d'autrui) ne peut à cette occasion qu'être inopérant puisque l'altérité mise en scène délègue toujours la figure d'un auteur-narrateur qui assure le maintien du récit. La (re)création du monde est en effet l'un des paramètres du projet leirisien. L'aveu était déjà explicite dans "Mors" puisque Leiris y soulignait de quelle manière la mort ne représentait pas seulement le constat angoissant de sa propre finitude. À l'encontre de ce point de vue, Leiris indiquait que la perte de la faculté de raconter signait la destruction du monde. L'unicité du sujet, à laquelle obéit la construction d'une singularité identitaire qui loge au cour du projet autobiographique, suppose en effet que le témoin de la mort d'autrui ne soit pas différent du sujet 
66

qui est voué à la disparition. Il y a là en effet un solipsisme narcissique des plus troublants qui ne cesse de parcourir l'œuvre de Leiris. J'ai fait valoir un peu plus tôt de quelle façon le projet autobiographique était régenté par cette toute-puissance du narrateur qui prétend raconter et situer rétrospectivement les faits et événements de sa vie. Comment ne pas y voir la violente expression d'une emprise dont l'écriture - comme maintien narcissique - est sans doute la figure privilégiée.

On retrouvera bien sûr de nombreux passages qui font jouer cette inscription de la dépressivité dans l'œuvre littéraire et le sentiment de diminution, de rétrécissement narcissique qui accompagne ce qui s'apparente à "un récit de disparition". En témoigne ce passage: "La tombe, qui a pour but de ramener aux dimensions normales de notre monde ce qui échappe à toutes dimensions. " ${ }^{23}$ Le titre même du dernier projet d'écriture, Les maisons vides, est sur ce point révélateur. La postérité est insupportable parce qu'elle implique qu'un autre, ayant le statut de témoin, puisse authentifier - et certifier du même coup - la validité de mon existence. D'où ce refus, réitéré par Leiris à la suite des remarques du jeune Liégois: il ne saurait être question de créer des personnages de papier, attributs de la fiction, alors même que le projet autobiographique inscrit la durabilité d'un soi qui pourrait échapper à la corruption. Mais les choses ne sont pas si simples puisque l'Unicité identitaire, au fondement de l'autobiographie, est d'emblée critiquée:

Ne pouvant faire coïncider l'Image que je fais de moi avec celle que les autres s'en font et ne pouvant même pas me fier à l'image apparemment objective que le miroir me renvoie puisque c'est ma vue à moi (certainement fallacieuse) que j'en ai, il me reste à me faufiler - non sans malaise - entre ces trois images. ${ }^{24}$

Tout se passe en effet comme si l'autobiographie maintenait par défaut cette illusion d'une complétude perceptive. Leiris le mentionne dans son Jourmal: l'authenticité ne peut être le fait du regard que l'on porte sur soi. Encore moins cette "authenticité" peut-elle être certifiée par la perception d'autrui. Il reste alors une solution médiane, certes insatisfaisante: l'apparence d'objectivité, que représente pour Leiris l'image du miroir, construit une repré- 
sentation qui est au coeur du projet autobiographique. Ne pouvant se complaire, encore moins se contenter de la représentation endopsychique qui peuple l'imaginaire, ou valider d'emblée le jugement d'autrui, il faudra trouver une insertion ternaire que représente peut-être ce miroir dont les propriétés apparaissent si singulières. À sa façon, ce miroir introduit une représentation objectivée de soi, mais par une étrange torsion qui fait de soi un Autre s'observant. Il y a bien sûr à cette occasion une mise en relation du champ spéculaire et de l'identité. Mais je crois que le propos de Leiris s'éloigne résolument de toute assomption perceptive. Si ce miroir offre une image de soi, c'est au prix d'un malaise qui est difficilement soutenable.

À cet égard, le projet autobiographique leirisien n'est pas sans rappeler l'aphanisis: évanouissement fantomatique, ou encore fading du sujet auquel Lacan fait référence pour mieux situer l'angoisse de disparition. Face à ce vide abyssal, il faut, c'est du moins le prétexte narratif soumis par le projet leirisien, écrire sans cesse. Pour mieux combler ce vide qui évoque la mort ou l'impensé, l'activité scripturale sera invoquée. On peut même ajouter que l'écriture est précisément ce "lieu" qui crée la figuration d'un vide dont la représentation peut devenir, par biais de l'élaboration autobiographique, une réalité supportable. Ce n'est pas un hasard si le chapitre de Fourbis auquel nous avons fait référence s'intitule précisément "Mors". Les références à ce "trou" qui inaugure l'activité symbolisante sont multiples: grottes, déclivités, anfractuosités, replis du corps féminin, trouée du rêve qui bouleverse la quotidienneté. Autant de représentations décisives de ce vide qui doit impérativement faire l'objet d'un discours transformant la non-pensée en absence. À l'exemple d'un Leiris qui écrit dans son Joumal:

Le pire, ce n'est plus de se dire qu'un jour rien ne sera pour vous, mais que - du même coup - rien n'aura été. Non seulement votre vie cessera mais - faute de votre mémoire capable de tant soit peu la récapituler - elle s'annulera (deviendra comme si rien ne s'était passé). Cette pensée est assurément déprimante à ressasser. [...] La confession n'est certes pas blanchiment, mais il se peut que dans une certaine mesure elle soit catharsis. (Dans la perspective que je trace ici, "formuler " n'a évidemment pas plus d'importance que le reste, mais le fait est là: si je dis, je vais mieux.) ${ }^{25}$

25 Ibid., p. 723-724. 